\title{
Predisposing factors for late mortality in heart transplant patients
}

\author{
Emyal Alyaydin ${ }^{1}$, Henryk Welp ${ }^{2}$, Holger Reinecke ${ }^{1}$, Izabela Tuleta ${ }^{1}$ \\ ${ }^{1}$ Department of Cardiology I, University of Muenster, Germany \\ ${ }^{2}$ Department of Cardiothoracic Surgery, University of Muenster, Germany
}

\begin{abstract}
Background: Because of the growing prevalence of terminal heart failure on the one hand and organ shortage on the other hand, an optimal care of heart transplant recipients based on the knowledge of potential risk factors not only early, but also in a long-term course after heart transplantation is of great importance. Therefore, the aim of the present study was to identify predisposing factors for late mortality in this patient collective.

Methods: Data from long-term heart transplant patients collected during follow-up visits in the current center were retrospectively analyzed. Clinical, laboratory, including immune monitoring and apparative examination results were studied with regard to all-cause mortality.

Results: One hundred and seventy-two patients after heart transplantation (mean: $13.2 \pm 6.4$ years) were divided into two groups: survivors $(n=133)$ and non-survivors $(n=39)$. In comparison with survivors, non-survivors were characterized by significantly more pronounced renal insufficiency with more frequent dialysis, anemia and worse functional status. Additionally, non-survivors obtained hearts from relevantly more obese donors. In a multivariate Cox regression analysis the following parameters were shown to be independent risk factors for increased mortality: CD4 percentage $<42 \%$, $C$-reactive protein $\geq 0.5 \mathrm{mg} / \mathrm{dL}$, presence of rejections requiring therapies in the past, onset of cardiac allograft vasculopathy $<5$ years following heart transplantation and no use of beta-blockers.

Conclusions: Low CD4+ cell percentages, sustained inflammation, relevant organ rejections, early onset of transplant vasculopathy and no use of beta-blockers are risk factors for higher mortality in a long-term follow-up after heart transplantation. (Cardiol J 2021; 28, 5: 746-757)
\end{abstract}

Key words: heart transplantation, immune monitoring, inflammation, organ rejection, transplant vasculopathy, beta-blocker therapy

\section{Introduction}

Heart failure is an increasing health disorder worldwide [1]. As ultima ratio therapy, heart transplantation (HTx) has been proven to be an effective method of treatment in selected groups of patients with terminal heart failure refractory to other treatments [2]. However, declining number of heart donors is a growing problem [3] which demands optimized management of the pre-, peri- and post-transplantation stages in order to effectively prolong organ function and reduce mortality. In contrast to numerous investigations on risk factors, potential complications and therapy options in the early phase following HTx $[4,5]$, there are relevantly few studies examining factors influencing survival many years after HTx [6-8]. Furthermore, the results of these studies cannot be extrapolated to a long-term survival as various factors and/or to a different extent may be associated with short- and long-term survival $[6,7,9,10]$. Some determinants such as malignancy, infection $[6,7,11]$, chronic rejection $[7,11]$, chronic allograft vasculopathy [11], idiopathic dilated cardiomyopa-

Address for correspondence: Izabela Tuleta, MD, $1^{\text {st }}$ Department of Cardiology, University of Muenster, Albert-Schweitzer-Campus 1, 48149 Muenster, Germany, tel: 0049/251/83-46760, fax: 0049/251/83-43204, e-mail: izat@gmx.de

Received: 8.12.2019 Accepted: 17.01.2020 Early publication date: 11.02.2020

This article is available in open access under Creative Common Attribution-Non-Commercial-No Derivatives 4.0 International (CC BY-NC-ND 4.0) license, allowing to download articles and share them with others as long as they credit the authors and the publisher, but without permission to change them in any way or use them commercially. 
thy and younger recipient age [8] were associated with late mortality following HTx. However, factors predicting mortality in long-term heart transplant survivors are still unknown in many cases.

Therefore, the aim of the present study was to define determinants favoring prolonged survival in heart transplant patients. Beyond clinical, standard laboratory and apparative findings, the focus was placed on results from immune monitoring which reflects present immune/inflammatory status of patients.

\section{Methods}

\section{Data collection}

The current retrospectively analyzed data were collected during the last control visits of heart transplant patients in the out-patient Department of Cardiology I at the University of Muenster. Of 483 patients who underwent HTx in the Department of Cardiothoracic Surgery at the University of Muenster between 1990 and 2018, 311 were excluded from the present study because of loss to follow-up $(\mathrm{n}=309)$ or HTx less than 3 years till follow-up $(n=2)$ (Fig. 1). During followup visits routine examinations including patient history, current complaints and medication, physical examination, assessment of functional capacity, arterial pressure, electrocardiogram, echocardiography and laboratory blood tests were conducted.

Standard laboratory blood tests consisted of measurements of electrolyte concentrations, renal and hepatic function, blood count, clotting parameters, inflammatory factors, N-terminalpro-B-type natriuretic peptide and levels of immunosuppressive drugs such as cyclosporine A (CsA), everolimus, tacrolimus, mycophenolate mofetil (MMF) and prednisolone depending on the current immunosuppressive medication. Additionally, immune monitoring encompassing total lymphocyte number, numbers and percentages of $\mathrm{CD} 4+, \mathrm{CD} 8+, \mathrm{CD} 19+$ and natural killer cells was performed. Further, in order to exclude current relevant viral or fungal infections respective molecular and serological examinations were conducted.

Inclusion criteria were HTx at least 3 years till follow-up and age $>18$ years at follow-up. Additionally, only patients in whom all the above mentioned parameters were determined within one visit at latest 1 year before the current assessment of the alive status or the date of death were enrolled in the study. All instable patients defined as patients presenting status demanding relevant changes in their current medication and/or

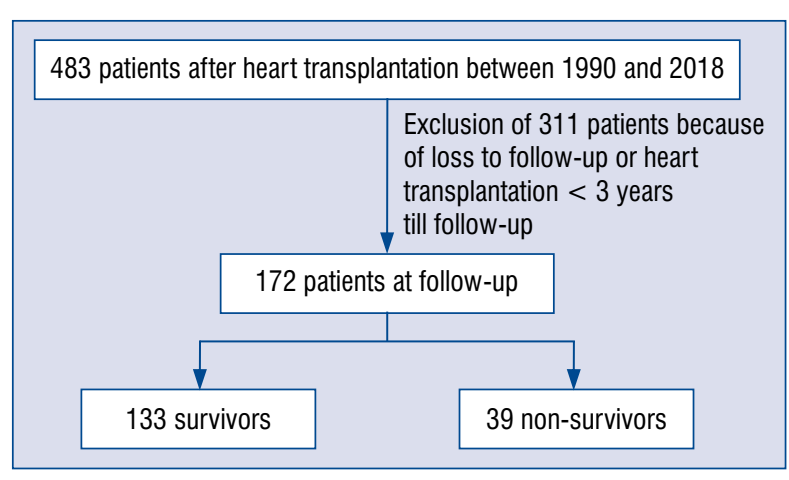

Figure 1. Flowchart of the study.

hospitalization were excluded from the study. Heart retransplantation was an additional exclusion criterion.

The study was performed according to the Declaration of Helsinki and the study protocol was approved by the local Ethics Committee of the University of Muenster.

\section{Statistical analysis}

Statistical analysis was conducted with IBM SPSS Statistics, version 25. Parametric values were expressed as means \pm standard deviation (SD). Differences between the means of two groups were assessed by the Student t-test. Comparisons between categorical variables were performed using the $\chi^{2}$ test. Two-tailed bivariate correlations were determined by the Pearson coefficient.

Potential risk factors for death were examined by the use of univariable and multivariable Cox proportional hazard function analyses. Variables showing a $p<0.05$ in the univariate Cox analysis were introduced in a multivariable Cox model and a stepwise selection process was used to select the final independent predictors of mortality. Survival in groups depending on the risk factors identified in the multivariable Cox analysis was compared with the log-rank test and was illustrated using the Kaplan-Meier curves. $\mathrm{P}<0.05$ was defined as statistically significant.

\section{Results}

\section{Patient characteristics}

One hundred and seventy-two patients at least 3 years after HTx (mean: $13.2 \pm 6.4$ years) were divided into two groups according to survival (survivors, $\mathrm{n}=133$; non-survivors, $\mathrm{n}=39$ ). There were no significant differences in demographics, including age, sex and body mass index, 
the presence of cardiovascular risk factors and extent of vasculopathy in coronary, carotid and peripheral arteries and left ventricular ejection fraction (LVEF) between the groups at follow-up. Parameters connected to HTx such as age at the time of HTx, time on the HTx list, urgency of the procedure and the frequency of the ventricular assist device use prior HTx did not relevantly differ between both groups. Non-survivors were characterized by significantly worse functional status according to the New York Heart Association (NYHA) Classification, earlier onset of transplant vasculopathy, more reduced right ventricular systolic function expressed as tricuspid annular plane systolic excursion, less frequent use of beta-blockers (BB) and more frequent use of platelet aggregation inhibitors and they were significantly more often on dialysis. Moreover, patients from the non-survivor group presented a tendency toward a higher average heart frequency and more frequent development of precarcinoma/ /carcinoma. The analysis of donor-associated factors demonstrated relevantly higher body weight in the non-survivor group. Most patients from both groups prior HTx suffered from heart failure as a result of dilated cardiomyopathy, followed by ischemic heart disease and congenital cardiomyopathy. In the group of non-survivors, the majority of patients died of malignancy and infections. The most frequent causes of death of donors comprised traumatic brain injury as well as subarachnoidal and intracerebral hemorrhage, without any relevant differences between the groups (Table 1).

Of note, when comparing patients with early onset of transplant vasculopathy to those with the late one, independently of survival status, patients with early onset of transplant vasculopathy were significantly older prior HTx $(55.3 \pm 8.3$ vs. $44.6 \pm$ \pm 15.1 years, $p=0.016$, respectively) and donors presented with tendentially higher weight $(82.3 \pm$ \pm 13.3 vs. $72.4 \pm 17.3 \mathrm{~kg}, \mathrm{p}=0.068$, respectively). Additionally, a comparison between patients with and without transplant vasculopathy irrespectively of the time of its onset and the survival status demonstrated some significant differences in clinical and laboratory parameters presented in Table 2.

\section{Immunosuppression and rejection}

The most frequent immunosuppressive therapy in both patient groups was a CsA-based one, followed by the everolimus- and tacrolimus-based therapies (Fig. 2A). The overwhelming number of survivors and non-survivors were on an additional therapy with low-dose prednisolone $(\mathrm{n}=104,78.2 \%$ vs. $\mathrm{n}=34,87.2 \%, \mathrm{p}=0.215)$ without differences in daily doses $(3.7 \pm 2.4 \mathrm{mg}$ vs. $4.3 \pm 2.2 \mathrm{mg}$, $\mathrm{p}=0.198$ ) between both groups.

The analysis of distinct subgroups of patients according to three main immunosuppressants: CsA, everolimus and tacrolimus, each combined with MMF showed in contrast to everolimus and tacrolimus significantly higher blood concentrations of $\mathrm{CsA}$ in the non-survivor versus survivor group (Fig. 2B-D).

Mycophenolate mofetil blood levels were similar both in survivors and non-survivors in the above mentioned three subgroups (Fig. 2B-D) and when comparing all survivors and non-survivors taking MMF, independently of the immunosuppressive co-medication $(2.2 \pm 1.8 \mathrm{ng} / \mathrm{mL}$ vs. $2.2 \pm 1.6 \mathrm{ng} / \mathrm{mL}$, $\mathrm{p}=0.962$, respectively).

Cellular-mediated rejections were classified into three grades according to the International Society of Heart and Lung Transplantation (ISHLT) grading system [12].

Total rejection number from HTx till the last follow-up was similar in both groups. There was a tendency toward higher frequency of therapy requiring rejections in the non-survivor group. Of note, the vast majority of therapy requiring rejections occurred within 2 years after HTx in both groups (Table 3 ).

\section{Immunological status and inflammation}

Immunological monitoring revealed significantly lower percentage of CD4+ cells among all lymphocytes in the blood in non-survivors versus survivors. Conversely, CD8 + cell portion was relevantly higher in this patient group. As a consequence, the $\mathrm{CD} 4 / \mathrm{CD} 8$ ratio tended to be lower in non-survivors. In contrast, there were no significant differences in the levels of other lymphocyte populations, such as CD19+ cells and natural killer cells between the groups.

Inflammatory response expressed as elevated leukocyte numbers, C-reactive protein (CRP) and interleukin (IL)-6 levels was significantly more pronounced in non-survivors (Table 3 ).

\section{Chronic kidney disease, heart failure and anemia}

Non-survivors were characterized by worse renal function expressed as a lower glomerular filtration rate and higher urea concentrations in the blood. Moreover, the diagnosis of anemia, defined according to the World Health Organization classification as hemoglobin $<13 \mathrm{~g} / \mathrm{dL}$ for men and hemoglobin $<12 \mathrm{~g} / \mathrm{dL}$ for women [13], 
Table 1. Clinical characteristics of patients at follow-up.

\begin{tabular}{|c|c|c|c|}
\hline Parameters & $\begin{array}{l}\text { Survivors (group 1) } \\
\qquad N=133\end{array}$ & $\begin{array}{l}\text { Non-survivors (group 2) } \\
\qquad N=39\end{array}$ & $\mathbf{P}$ \\
\hline Age [years] & $59.2 \pm 15.4$ & $58.5 \pm 16.7$ & 0.821 \\
\hline Male sex & $96(72.2 \%)$ & $32(82.1 \%)$ & 0.214 \\
\hline Body mass index [kg/m²] & $26.2 \pm 5.4$ & $25.1 \pm 5.6$ & 0.265 \\
\hline Age at $\mathrm{HTx}$ [years] & $44.7 \pm 15.0$ & $47.6 \pm 14.9$ & 0.273 \\
\hline Time on HTx transplant list [months] & $288.3 \pm 326.1$ & $286.7 \pm 350.3$ & 0.980 \\
\hline High urgency & $51(38.3 \%)$ & $14(35.9 \%)$ & 0.782 \\
\hline VAD prior HTx & $47(35.3 \%)$ & $12(30.8 \%)$ & 0.597 \\
\hline Follow-up after HTx [years] & $14.0 \pm 6.5$ & $10.4 \pm 5.2$ & $0.002 *$ \\
\hline \multicolumn{4}{|l|}{ Clinical examination } \\
\hline NYHA > 1 & $55(41.4 \%)$ & $26(66.7 \%)$ & $0.005^{*}$ \\
\hline Systolic BP [mmHg] & $126 \pm 20$ & $121 \pm 15$ & 0.158 \\
\hline Diastolic BP [mmHg] & $79 \pm 11$ & $76 \pm 9$ & 0.088 \\
\hline Heart frequency & $82 \pm 13$ & $86 \pm 13$ & 0.099 \\
\hline \multicolumn{4}{|l|}{ Echocardiography } \\
\hline LVEF [\%] & $55.7 \pm 7.3$ & $55.9 \pm 11.5$ & 0.917 \\
\hline TAPSE [mm] & $16.6 \pm 4.2$ & $14.8 \pm 4.4$ & $0.019 *$ \\
\hline \multicolumn{4}{|l|}{ Cardiovascular risk factors } \\
\hline Arterial hypertension & $107(80.5 \%)$ & $31(79.5 \%)$ & 0.894 \\
\hline Diabetes mellitus & $33(24.8 \%)$ & $13(33.3 \%)$ & 0.290 \\
\hline Hypercholesterolemia & $117(88.0 \%)$ & $35(89.7 \%)$ & 0.761 \\
\hline Nicotine abuse: & & & 0.532 \\
\hline Never smoker & $117(88.0 \%)$ & $32(82.1 \%)$ & \\
\hline Current smoker & $5(3.8 \%)$ & $3(7.7 \%)$ & \\
\hline Former smoker & $11(8.3 \%)$ & $4(10.3 \%)$ & \\
\hline Transplant vasculopathy & $53(39.8 \%)$ & $14(35.9 \%)$ & 0.656 \\
\hline $\begin{array}{l}\text { Transplant vasculopathy requiring } \\
\text { invasive therapy }\end{array}$ & $27(20.3 \%)$ & $11(28.2 \%)$ & 0.295 \\
\hline $\begin{array}{l}\text { Onset of transplant vasculopathy } \\
<5 \text { years after HTx }\end{array}$ & $5(3.8 \%)$ & $7(17.9 \%)$ & $0.002 *$ \\
\hline CAD/PAD & $18(13.5 \%)$ & $8(20.5 \%)$ & 0.285 \\
\hline Dialysis & $19(14.3 \%)$ & $12(30.8 \%)$ & $0.019 *$ \\
\hline Precarcinoma/carcinoma & $35(26.3 \%)$ & $15(38.5 \%)$ & 0.142 \\
\hline Obstructive or restrictive lung diseases & $21(15.8 \%)$ & $11(28.2 \%)$ & 0.080 \\
\hline \multicolumn{4}{|l|}{ Cardiovascular medication } \\
\hline Beta-blocker & $78(58.6 \%)$ & $15(38.5 \%)$ & $0.026^{*}$ \\
\hline Calcium channel inhibitor: & & & 0.754 \\
\hline Diltiazem & $44(33.1 \%)$ & $11(28.2 \%)$ & \\
\hline Dihydropyridine & $33(24.8 \%)$ & $9(23.1 \%)$ & \\
\hline Ivabradine & $6(4.5 \%)$ & $2(5.1 \%)$ & 0.872 \\
\hline ACE inhibitor/AT1R antagonist & $83(62.4 \%)$ & $18(46.2 \%)$ & 0.070 \\
\hline Statin & $109(82.0 \%)$ & $31(79.5 \%)$ & 0.728 \\
\hline Pravastatin equivalent dose & $43.2 \pm 47.9$ & $37.4 \pm 40.6$ & 0.493 \\
\hline Diuretics: & & & 0.258 \\
\hline Thiazide & $2(1.5 \%)$ & $1(2.6 \%)$ & \\
\hline Loop diuretics & $69(51.9 \%)$ & $16(41.0 \%)$ & \\
\hline Aldosterone antagonists & $2(1.5 \%)$ & $1(2.6 \%)$ & \\
\hline Combined diuretics & $11(8.3 \%)$ & $8(20.5 \%)$ & \\
\hline Platelet aggregation inhibitors: & & & $0.048^{*}$ \\
\hline ASS & $33(24.8 \%)$ & $12(30.8 \%)$ & \\
\hline Clopidogrel & $24(18.0 \%)$ & $2(5.1 \%)$ & \\
\hline Combined ASS and clopidogrel & $7(5.3 \%)$ & $6(15.4 \%)$ & \\
\hline Oral anticoagulation & $19(14.3 \%)$ & $7(17.9 \%)$ & 0.320 \\
\hline
\end{tabular}


Table 1. Clinical characteristics of patients at follow-up.

\begin{tabular}{|c|c|c|c|}
\hline Parameters & $\begin{array}{l}\text { Survivors (group 1) } \\
\qquad N=133\end{array}$ & $\begin{array}{l}\text { Non-survivors (group 2) } \\
\qquad N=39\end{array}$ & $\mathbf{P}$ \\
\hline Etiology of heart failure prior HTx & & & 0.312 \\
\hline Dilated cardiomyopathy & $63(47.4 \%)$ & $14(35.9 \%)$ & \\
\hline Ischemic cardiomyopathy & $46(34.6 \%)$ & $19(48.7 \%)$ & \\
\hline Congenital cardiomyopathy & $10(7.5 \%)$ & $2(5.1 \%)$ & \\
\hline Arrhythmogenic right ventricular dysplasia & $3(2.3 \%)$ & $0(0.0 \%)$ & \\
\hline Postpartum cardiomyopathy & $1(0.8 \%)$ & $0(0.0 \%)$ & \\
\hline Non-compaction cardiomyopathy & $2(1.5 \%)$ & $0(0.0 \%)$ & \\
\hline Hypertrophic cardiomyopathy & $3(2.3 \%)$ & $1(2.6 \%)$ & \\
\hline Myocarditis-related cardiomyopathy & $2(1.5 \%)$ & $2(5.1 \%)$ & \\
\hline Valvular cardiomyopathy & $3(2.3 \%)$ & $0(0.0 \%)$ & \\
\hline Toxic cardiomyopathy & $0(0.0 \%)$ & $1(2.6 \%)$ & \\
\hline \multicolumn{4}{|l|}{ Causes of death } \\
\hline Malignant tumor & & $8(20.5 \%)$ & \\
\hline Sepsis & & $6(15.4 \%)$ & \\
\hline Pneumonia & & $4(10.3 \%)$ & \\
\hline Sudden cardiac death & & $4(10.3 \%)$ & \\
\hline Cardiogenic shock & & $3(7.7 \%)$ & \\
\hline Renal failure & & $2(5.1 \%)$ & \\
\hline Chronic transplant vasculopathy & & $2(5.1 \%)$ & \\
\hline Vascular dementia & & $1(2.6 \%)$ & \\
\hline Ascending aortic aneurysm & & $1(2.6 \%)$ & \\
\hline Hemorrhagic esophagitis & & $1(2.6 \%)$ & \\
\hline Unknown & & $7(17.9 \%)$ & \\
\hline \multicolumn{4}{|l|}{ Donor parameters } \\
\hline Age [years] & $31.0 \pm 13.4$ & $31.1 \pm 13.9$ & 0.949 \\
\hline Body weight $[\mathrm{kg}]$ & $71.6 \pm 17.0$ & $78.2 \pm 17.2$ & $0.048^{*}$ \\
\hline Body height $[\mathrm{cm}]$ & $172.8 \pm 16.8$ & $174.5 \pm 22.8$ & 0.615 \\
\hline Sex (male) & $71(53.4 \%)$ & $22(56.4 \%)$ & 0.859 \\
\hline Causes of death: & & & 0.148 \\
\hline Traumatic brain injury & $47(35.3 \%)$ & $11(28.2 \%)$ & \\
\hline Subarachnoidal hemorrhage & $28(21.1 \%)$ & $4(10.3 \%)$ & \\
\hline Intracerebral hemorrhage & $16(12.0 \%)$ & $7(17.9 \%)$ & \\
\hline Meningitis & $4(3.0 \%)$ & $0(0.0 \%)$ & \\
\hline Cerebral ischemia & $3(2.3 \%)$ & $5(12.8 \%)$ & \\
\hline Intracranial aneurysm & $2(1.5 \%)$ & $1(2.6 \%)$ & \\
\hline Cerebral edema & $2(1.5 \%)$ & $0(0.0 \%)$ & \\
\hline Hypoxic brain injury & $2(1.5 \%)$ & $1(2.6 \%)$ & \\
\hline Polytrauma & $2(1.5 \%)$ & $2(5.1 \%)$ & \\
\hline Gun shot skull injury & $2(1.5 \%)$ & $2(5.1 \%)$ & \\
\hline Cardiovascular arrest & $1(0.8 \%)$ & $0(0.0 \%)$ & \\
\hline Status epilepticus & $1(0.8 \%)$ & $0(0.0 \%)$ & \\
\hline Strangulation & $1(0.8 \%)$ & $1(2.6 \%)$ & \\
\hline Subdural hematoma & $1(0.8 \%)$ & $0(0.0 \%)$ & \\
\hline Intoxication & $1(0.8 \%)$ & $0(0.0 \%)$ & \\
\hline Fetal death & $0(0.0 \%)$ & $1(2.6 \%)$ & \\
\hline Unknown & $20(15.0 \%)$ & $4(10.3 \%)$ & \\
\hline Donor recipient sex match/mismatch & & & 0.470 \\
\hline No data & $23(17.3 \%)$ & $5(12.8 \%)$ & \\
\hline Match & $84(63.2 \%)$ & $25(64.1 \%)$ & \\
\hline Male $\rightarrow$ female & $17(12.8 \%)$ & $8(20.5 \%)$ & \\
\hline Female $\rightarrow$ male & $9(6.8 \%)$ & $1(2.6 \%)$ & \\
\hline
\end{tabular}

Data are presented as a mean \pm standard deviation or number (percentage); ${ }^{*} \mathrm{p}<0.05$ (significant); ACE - angiotensin-converting-enzyme; ASS - acetylsalicylic acid; AT1R — angiotensin II type 1 receptor; BP — blood pressure; CAD — cerebral artery disease; HTx - heart transplantation; LVEF — left ventricular ejection fraction; NYHA - New York Heart Association; PAD - peripheral artery disease; TAPSE — tricuspid annular plane systolic excursion; VAD - ventricular assist device 
Table 2. Differences in clinical and laboratory parameters between patients with and without transplant vasculopathy at follow-up.

\begin{tabular}{lccc}
\hline Parameters & $\begin{array}{c}\text { Transplant vasculopathy } \\
(\mathbf{n}=\mathbf{1 0 5})\end{array}$ & $\begin{array}{c}\text { No transplant vasculopathy } \\
(\mathbf{n}=\mathbf{6 7})\end{array}$ & $\mathbf{P}$ \\
\hline Recipient BMI $\left[\mathrm{kg} / \mathrm{m}^{2}\right]$ & $27.1 \pm 5.9$ & $25.3 \pm 5.0$ & $0.032^{*}$ \\
Donor weight $[\mathrm{kg}]$ & $77.0 \pm 15.3$ & $70.8 \pm 18.0$ & $0.032^{*}$ \\
Donor age [years] & $35.4 \pm 12.3$ & $28.2 \pm 13.5$ & $0.001^{*}$ \\
Urea [mg/dL] & $37.1 \pm 21.0$ & $30.5 \pm 17.4$ & $0.028^{*}$ \\
Follow-up after HTx [years] & $15.6 \pm 5.7$ & $11.7 \pm 6.3$ & $<0.001^{*}$ \\
Diabetes mellitus & $24(35.8 \%)$ & $22(21.0 \%)$ & $0.032^{*}$ \\
Osteoporosis & $11(16.4 \%)$ & $5(4.8 \%)$ & $0.010^{*}$ \\
Platelet aggregation inhibitors & $46(68.7 \%)$ & $38(36.2 \%)$ & $<0.001^{*}$ \\
Oral anticoagulation & $15(22.4 \%)$ & $11(10.5 \%)$ & $0.032^{*}$ \\
Diuretics & $49(73.1 \%)$ & $61(58.1 \%)$ & $0.045^{*}$ \\
\hline
\end{tabular}

Data are presented as a mean \pm standard deviation or number (percentage); ${ }^{*} \mathrm{p}<0.05$ (significant); BMI — body mass index; HTx - heart transplantation

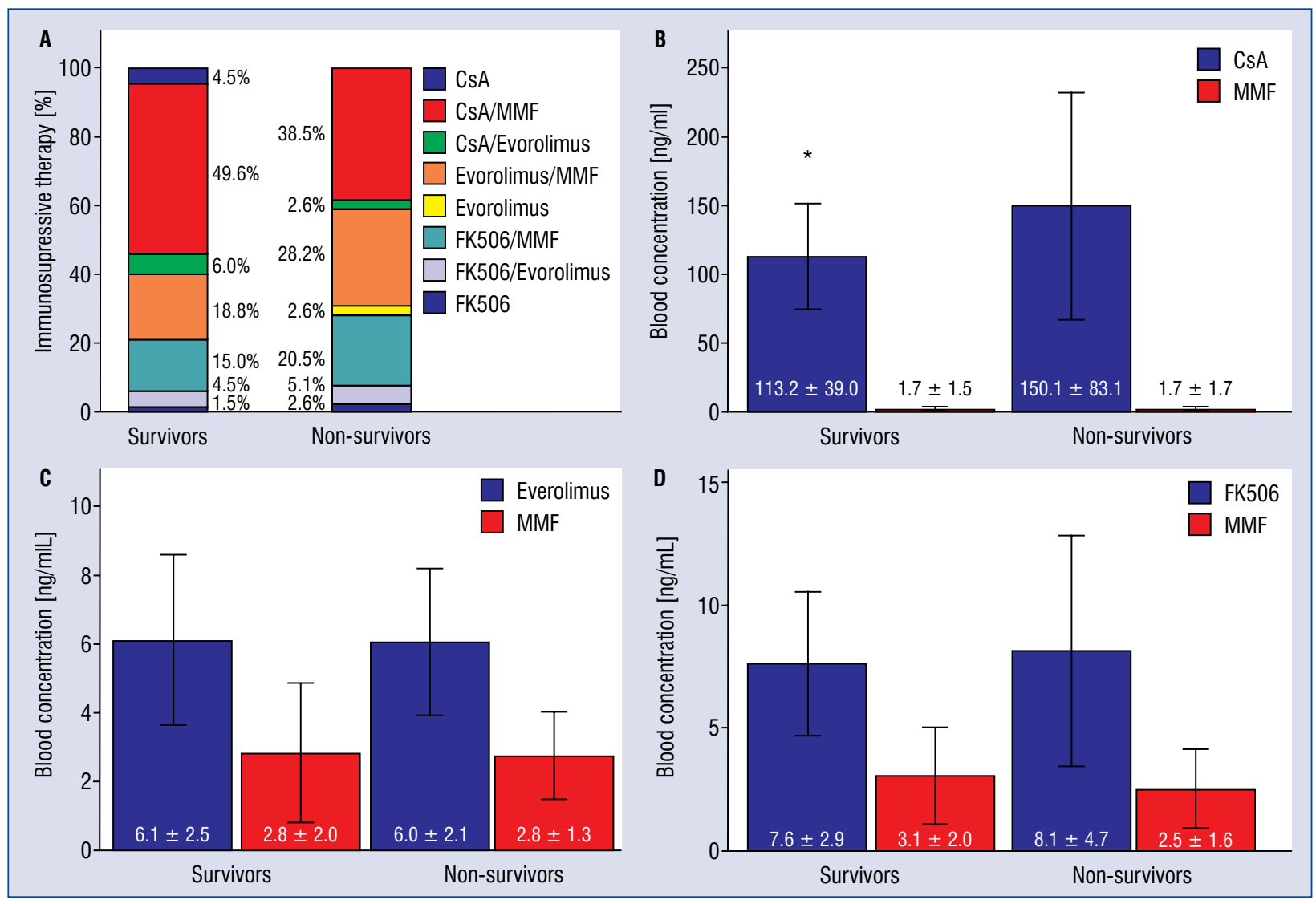

Figure 2. A-D. Immunosuppressive therapy in survivors and non-survivors; CsA - cyclosporine A; MMF - mycophenolate mofetil; FK506 - tacrolimus; ${ }^{*} p<0.05$ (significant).

was significantly more frequent in non-survivors. The morphological and biochemical analysis of erythrocytes showed macrocytic and hypochromic anemia.
$\mathrm{N}$-terminal-pro-B-type natriuretic peptide values were significantly higher in non-survivors, although echocardiographically estimated LVEF did not differ between the groups (Table 4). 
Table 3. Number and severity of rejections since heart transplantation till the last follow-up as well as immunological and inflammatory factors at follow-up in survivors and non-survivors.

\begin{tabular}{|c|c|c|c|}
\hline & $\begin{array}{l}\text { Survivors (group 1) } \\
\qquad N=133\end{array}$ & $\begin{array}{l}\text { Non-survivors (group 2) } \\
\qquad N=39\end{array}$ & $\mathbf{P}$ \\
\hline Rejections & $73(54.9 \%)$ & $24(61.5 \%)$ & 0.461 \\
\hline Rejection stage: & & & 0.299 \\
\hline 1 & $31(23.3 \%)$ & $7(17.9 \%)$ & \\
\hline 2 & $39(29.3 \%)$ & $14(35.9 \%)$ & \\
\hline 3 & $3(2.3 \%)$ & $3(7.7 \%)$ & \\
\hline Rejections requiring therapy & $45(33.8 \%)$ & $18(46.2 \%)$ & 0.160 \\
\hline $\begin{array}{l}\text { Rejections requiring therapy under } 24 \text { months } \\
\text { after heart transplantation }\end{array}$ & $40(88.9 \%)$ & $13(72.2 \%)$ & 0.102 \\
\hline Leukocytes (G/L) & $7.3 \pm 2.5$ & $8.2 \pm 2.7$ & $0.048^{*}$ \\
\hline Lymphocytes [cells $/ \mu \mathrm{L}$ ] & $1542.6 \pm 687.3$ & $1519.1 \pm 960.0$ & 0.865 \\
\hline CD3 [cells $/ \mu \mathrm{L}]$ & $1217.7 \pm 634.6$ & $1212.8 \pm 894.2$ & 0.969 \\
\hline CD3 [\%] & $77.3 \pm 11.3$ & $78.0 \pm 11.3$ & 0.731 \\
\hline CD4 [cells/ $\mu \mathrm{L}]$ & $700.1 \pm 325.2$ & $616.4 \pm 400.0$ & 0.183 \\
\hline CD4 [\%] & $46.2 \pm 10.9$ & $42.0 \pm 11.8$ & $0.042 *$ \\
\hline CD8 [cells/ $\mu \mathrm{L}]$ & $480.7 \pm 427.7$ & $569.4 \pm 545.4$ & 0.287 \\
\hline CD8 [\%] & $28.7 \pm 13.5$ & $34.2 \pm 16.3$ & $0.035^{*}$ \\
\hline CD4/CD8 & $2.3 \pm 2.0$ & $1.7 \pm 1.2$ & 0.087 \\
\hline CD19 [cells $/ \mu \mathrm{L}]$ & $89.7 \pm 79.6$ & $73.1 \pm 86.3$ & 0.262 \\
\hline CD19 [\%] & $6.0 \pm 5.0$ & $4.5 \pm 3.5$ & 0.080 \\
\hline Natural killers [cells $/ \mu \mathrm{L}$ ] & $224.9 \pm 151.1$ & $214.1 \pm 156.4$ & 0.696 \\
\hline Natural killers [\%] & $16.0 \pm 10.2$ & $15.8 \pm 10.3$ & 0.939 \\
\hline Interleukin-6 [pg/mL] & $9.5 \pm 9.8$ & $14.2 \pm 20.3$ & $0.047^{*}$ \\
\hline C-reactive protein [mg/dL] & $0.8 \pm 0.9$ & $1.7 \pm 2.6$ & $0.001^{*}$ \\
\hline
\end{tabular}

All percentages are expressed as the number of distinct lymphocyte subsets divided by the number of all lymphocytes multiplied by $100 \%$. Data are presented as a mean \pm standard deviation or number (percentage); ${ }^{*} p<0.05$ (significant)

Table 4. Laboratory parameters connected to the heart and renal function as well as red blood cell parameters in survivors and non-survivors.

\begin{tabular}{lccc}
\hline & $\begin{array}{c}\text { Survivors (group 1) } \\
\mathbf{N}=\mathbf{1 3 3}\end{array}$ & $\begin{array}{c}\text { Non-survivors (group 2) } \\
\mathbf{N}=39\end{array}$ \\
\hline NT-proBNP $[\mathrm{pg} / \mathrm{mL}]$ & $3068.0 \pm 6172.2$ & $8397.6 \pm 11303.3$ & $<0.001^{*}$ \\
GFR $\left[\mathrm{mL} / \mathrm{min} / 1.73 \mathrm{~m}^{2}\right]$ & $46.4 \pm 24.1$ & $34.8 \pm 22.0$ & $0.008^{*}$ \\
Urea $[\mathrm{mg} / \mathrm{dL}]$ & $31.1 \pm 17.4$ & $39.9 \pm 23.0$ & $0.011^{*}$ \\
Erythrocytes [T/L] & $4.5 \pm 0.7$ & $4.2 \pm 0.7$ & $0.039^{*}$ \\
Hemoglobin [g/dL] & $12.7 \pm 1.8$ & $12.0 \pm 1.8$ & $0.041^{*}$ \\
Hematocrit [\%] & $39.2 \pm 5.2$ & $37.7 \pm 5.3$ & 0.116 \\
Mean corpuscular volume [fL] & $88.1 \pm 5.7$ & $90.1 \pm 7.5$ & 0.077 \\
Mean corpuscular hemoglobin $[\mathrm{pg}]$ & $28.6 \pm 2.1$ & $28.8 \pm 2.7$ & 0.670 \\
Mean corpuscular hemoglobin concentration $[\mathrm{g} / \mathrm{dL}]$ & $32.4 \pm 1.2$ & $31.9 \pm 1.3$ & $0.022^{*}$ \\
\hline
\end{tabular}

Data are presented as a mean \pm standard deviation; ${ }^{*} p<0.05$ (significant); GFR - glomerular filtration rate; NT-proBNP $-\mathrm{N}$-terminal-pro-B-type natriuretic peptide 


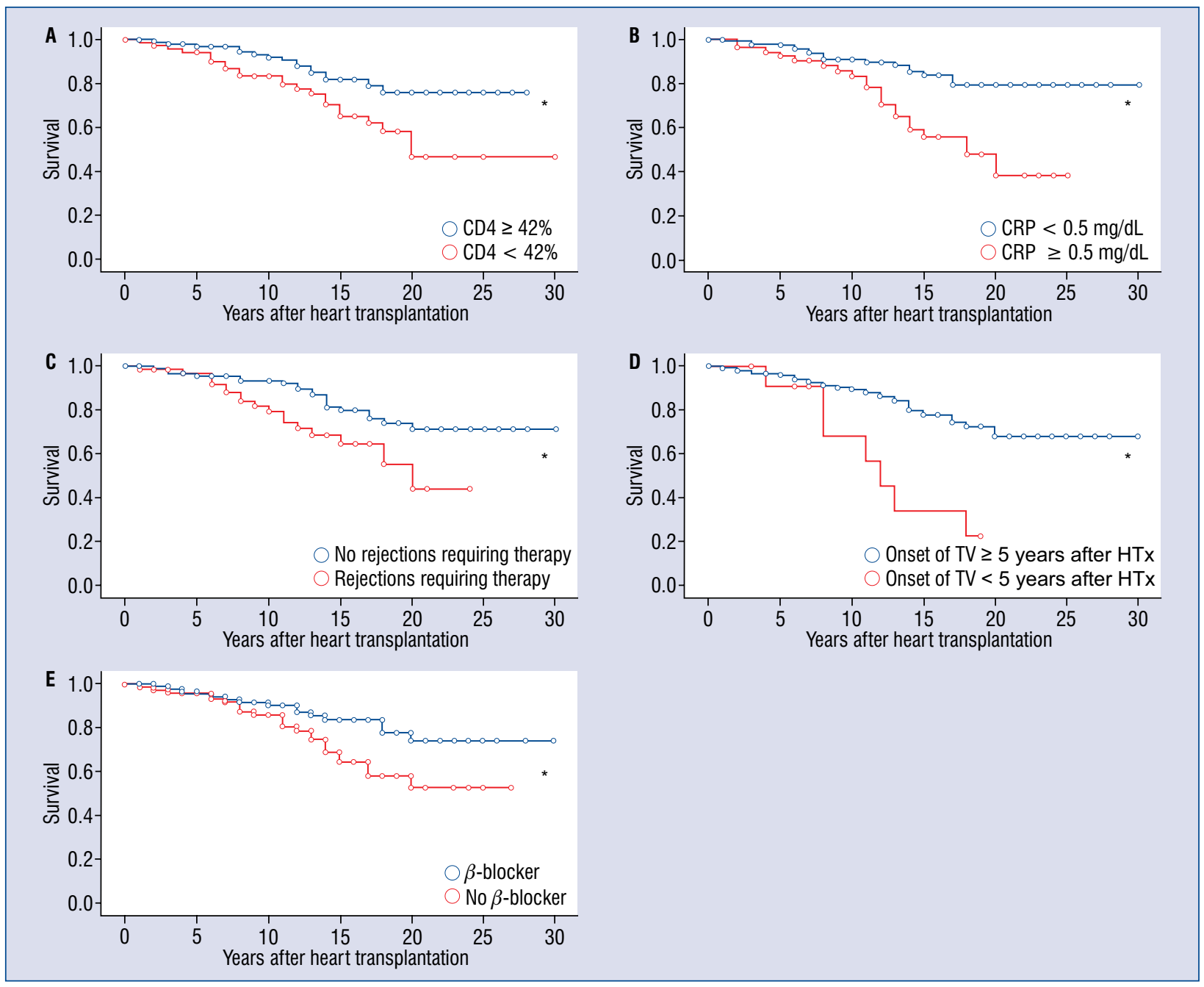

Figure 3. Kaplan-Meier curves show differences in survival of patients after heart transplantation (HTx). Factors positively influencing survival in patients after HTx; A. CD4 percentage equal or above $42 \%$; B. C-reactive protein (CRP) blood concentrations under $0.5 \mathrm{mg} / \mathrm{dL}$; $\mathbf{C}$. No rejections requiring therapy; $\mathbf{D}$. Late onset of transplant vasculopathy (TV) 5 years or more following HTx; E. The use of beta-blockers; ${ }^{*} \mathrm{p}<0.05$ (significant).

\section{Univariate and multivariate Cox analyses}

In the multivariate Cox analysis only five determinants among other potential risk factors tested in the univariate Cox analysis such as NYHA stages II and III vs. I ( $\mathrm{p}=0.033)$ and dialysis $(\mathrm{p}=0.108)$ showed a clear, statistically significant negative influence on the survival of heart transplant patients. These were: percentage of $\mathrm{CD} 4+$ cells $<42 \%$ (hazard ratio [HR]: 1.984 , confidence interval [CI]: $1.020-3.859, \mathrm{p}=0.044), \mathrm{CRP} \geq 0.5$ $\mathrm{mg} / \mathrm{dL}$ (HR: 3.422, CI: 1.767-6.626, p < 0.001), rejections requiring therapies (HR: 2.236 , CI: $1.157-4.319, \mathrm{p}=0.017$ ), early onset of transplant vasculopathy $<5$ years following HTx (HR: 2.741, CI: $1.145-6.558, \mathrm{p}=0.024$ ) and no use of BB (HR: 2.358, CI: 1.194-4.656, $p=0.013$; Fig. 3).

\section{Correlations}

The above mentioned five factors influencing survival in the multivariate analysis were correlated with other measures. Significant correlations are depicted in Table 5.

\section{Discussion}

The present study has shown that the main factors influencing survival in the long-term follow-up after HTx were connected to immunomodulation/ /inflammation, severe organ rejections, early onset of transplant vasculopathy and drug therapy.

Specifically, higher percentages of CD4+ cells were associated with significantly longer survival. $\mathrm{CD} 4+$ cells are central cells in the rejection pro- 
Table 5. Statistically significant correlations between factors influencing survival in the multivariate analysis and other measures.

\begin{tabular}{|c|c|}
\hline Correlations & $\mathbf{P}$ \\
\hline \multicolumn{2}{|l|}{ CD4\% } \\
\hline \multicolumn{2}{|l|}{ Negative correlations: } \\
\hline $\mathrm{CD} 8 \%, \mathrm{R}=-0.635$ & $<0.001^{*}$ \\
\hline Lymphocyte number, $R=-0.173$ & $0.023^{*}$ \\
\hline Natural killer cells, $R=-0.226$ & $0.003^{*}$ \\
\hline C-reactive protein, $R=-0.153$ & $0.045^{*}$ \\
\hline \multicolumn{2}{|l|}{ CRP } \\
\hline \multicolumn{2}{|l|}{ Negative correlations: } \\
\hline$C D 4 \%, R=-0.153$ & $0.045^{*}$ \\
\hline$C D 19, R=-0.154$ & $0.044^{*}$ \\
\hline Erythrocytes, $R=-0.276$ & $<0.001^{*}$ \\
\hline Hemoglobin, $R=-0.281$ & $<0.001^{*}$ \\
\hline Hematocrit, $R=-0.241$ & $0.002^{*}$ \\
\hline $\mathrm{MCHC}, \mathrm{R}=-0.250$ & $0.001^{*}$ \\
\hline GFR, $R=-0.277$ & $<0.001^{*}$ \\
\hline \multicolumn{2}{|l|}{ Positive correlations: } \\
\hline$C D 8 \%, R=0.187$ & $0.014^{*}$ \\
\hline Prednisolone, $R=0.267$ & $<0.001^{*}$ \\
\hline Dialysis, $R=0.312$ & $<0.001^{*}$ \\
\hline$C A D / P A D, R=0.219$ & $0.004^{*}$ \\
\hline NT-proBNP, $R=0.450$ & $<0.001^{*}$ \\
\hline Interleukin-6, $R=0.373$ & $<0.001^{*}$ \\
\hline $\mathrm{MCV}, \mathrm{R}=0.176$ & $0.021 *$ \\
\hline Diuretics, $R=0.166$ & $0.030 *$ \\
\hline \multicolumn{2}{|l|}{ Rejections requiring therapy } \\
\hline \multicolumn{2}{|l|}{ Positive correlations: } \\
\hline Rejection grade, $R=0.882$ & $<0.001^{*}$ \\
\hline \multicolumn{2}{|c|}{ Transplant vasculopathy under 5 years after HTx } \\
\hline \multicolumn{2}{|l|}{ Positive correlations: } \\
\hline Age at $H T x, R=0.184$ & $0.016^{*}$ \\
\hline \multicolumn{2}{|l|}{ Beta-blocker } \\
\hline \multicolumn{2}{|l|}{ Negative correlations: } \\
\hline Heart frequency, $R=-0.274$ & $<0.001^{*}$ \\
\hline Diastolic blood pressure, $R=-0.165$ & $0.030^{*}$ \\
\hline
\end{tabular}

${ }^{*} \mathrm{p}<0.05$ (significant); CAD - cerebral artery disease; GFR - glomerular filtration rate; $\mathrm{HTx}$ - heart transplantation; MCV - mean corpuscular volume; $\mathrm{MCHC}$ - mean corpuscular hemoglobin concentration, NT-proBNP - N-terminal-pro-B-type natriuretic peptide; $\mathrm{PAD}$ - peripheral artery disease

cess [14]. In the early stage after HTx these cells may mediate rejection responses against donor tissue causing cardiovascular damage with subsequent organ failure. Therefore, the consequent immunosuppressive therapy is mandatory to preserve normal structure and function of the transplanted heart. In contrast, aggressive immunosuppression in a long-term course is not needed because of a slowly developing immune tolerance. The present findings indicate that lower percentages of CD4+ cells may be associated with enhanced mortality many years after HTx. It may be related to two main reasons. On the one hand, reduced CD4 levels result mostly from higher blood concentrations of immunosuppressive drugs [15]. In the current study, subgroup analysis according to the immunosuppressive medication demonstrated that non-survivors treated with CsA-based immunosuppressive therapy had relevantly higher CsA blood concentrations compared to the corresponding survivor subgroup. As CsA therapy is connected to many side-effects, e.g. renal insufficiency [16] which indeed was more pronounced in the nonsurvivor group, relatively low $\mathrm{CD} 4+$ cell percentages could be seen as an indicator of an overly intensive drug therapy. However, in the subgroup of patients with tacrolimus-based immunosuppressive treatment, there were no significant differences in tacrolimus blood concentrations in spite of significantly higher CD4+ cell levels in survivors. In the subgroup with everolimus as a main immunosuppressant there were no relevant differences in everolimus or $\mathrm{CD} 4$ concentrations between survivors and non-survivors. No significant correlations were found between the percentage of $\mathrm{CD} 4+$ cells and the levels of the immunosuppressive medication used. Moreover, although it is known that low blood levels of CD4+ cells may lead to the renal failure [17] and vice versa reduced CD4 + cell percentage may be the result of an impaired renal function [18], no relevant correlations were demonstrated for $\mathrm{CD} 4$ percentage and renal function. This emphasizes the high complexity of an immune answer indicating individual response of the body to immunosuppressive therapy, renal function and/or additional mechanisms influencing CD4+ cell levels in the blood. This result shows that monitoring of drug concentrations and/or of renal function in the blood may be not sufficient to assess current immunological status and thus its impact on the body [19]. The other explanation of low CD4+ cell-associated mortality in the current collective of patients could be the creation of a prolonged subclinical immunosuppressive state with susceptibility to the development of sustained inflammation, infections and tumor diseases. Indeed, increased leukocyte numbers were found to be significant, as well as CRP and IL-6 levels in non-survivors. Additionally, CRP correlated negatively with CD4+ cell percentages. Furthermore, CRP was another 
factor that predicted higher mortality in a multivariate analysis. Chronic inflammation, indicated by increased CRP levels is a known independent factor for cardiovascular and all-cause mortality [20]. Prolonged immunosuppressive state may have also influenced a tendency toward increased prevalence of precarcinoma/tumor diseases [21]. This could have also emerged from the side-effects of immunosuppressive drugs [21]. In the present study patients were free of infections based on anamnesis, physical examination, laboratory and microbiological tests at follow-up. However, occult infections, not routinely tested in the current laboratory, could not be excluded. The results from the mortality data support the above hypothesis as the main causes of death in our patient population were malignant tumors and infections.

Furthermore, it has been shown that $\mathrm{CD} 4$ cell lymphopenia may accelerate the development of cardiovascular atherosclerotic complications in renal transplant recipients [22], which augments mortality risk. In the current study there were no significant differences in the occurrence and severity of transplant vasculopathy between survivors and non-survivors despite relevantly different levels of $\mathrm{CD} 4+$ cells. However, data concerning $\mathrm{CD} 4+$ cell blood concentrations in the past as atherosclerotic lesions were detected and invasively treated are missing, so that this issue cannot be covered by the present study.

The next parameter which was shown to be relevant in the context of survival was the presence of rejections requiring therapies in the past. In contrast, weaker rejections without the need for drug therapy were not of relevance regarding mortality. As gross of rejections requiring therapies occurred early after HTx, this points to a dual role of $\mathrm{CD} 4+$ cells in the outcome depending on the time course after HTx. Whereas an intense suppression of immunological response involving activation of $\mathrm{CD} 4+$ cells prevents rejections and thus organ failure early after $\mathrm{HTx}$, the continuation of a strong elimination of CD4+ cells years following HTx may contribute to a higher mortality. Therefore, continuous adjustments of immunosuppressive therapy strategies as well as close monitoring of immunological status in the blood are important actions at every time stage after HTx.

Immunological status, together with the sideeffects of drug therapy, cardiovascular risk factors and donor and recipient demographics at the time point of HTx procedure influence the onset of transplant vasculopathy [23]. Interestingly, the present study showed that there were no significant differences in the prevalence of transplant vasculopathy between survivors and non-survivors at the time point of the last follow-up. The presence of transplant vasculopathy was also not a factor influencing mortality in the Cox analysis. Patients with transplant vasculopathy were characterized by elevated cardiovascular risk factors such as diabetes mellitus, renal insufficiency, they were more obese, had longer heart transplant from older and more obese donors and differed from nontransplant vasculopathy patients in cardiovascular medication. Since survivors presented significantly less frequent transplant vasculopathy less than 5 years after HTx compared with non-survivors in this study, this finding suggests that, not just the presence of transplant vasculopathy is critical for survival, but much more the time point of its development. It is known that the immune mechanisms and the influence of immunomodulating drug therapy prevail in the development of transplant vasculopathy at early stages, whereas classical cardiovascular risk factors may play a greater role later in the time course [23]. In the present patient population typical cardiovascular risk factors at follow-up were equally distributed across both groups. Patients with early onset of transplant vasculopathy were significantly older at the HTx and the hearts were derived from tendentially more obese donors. This suggests that the early occurrence of transplant vasculopathy and thus higher risk of longer duration of transplant vasculopathy and the pathomechanisms determining its onset, including donor- and recipient-associated factors may influence long-term outcome following HTx.

The last factor presented to influence survival of long-term heart transplant patients was the therapy with BB in this study. Survivors obtained significantly more frequent $\mathrm{BB}$ treatment than nonsurvivors. As a consequence, the average heart frequency tended to be lower in these patients. Beta-blocker is a known drug reducing mortality in patients with systolic heart failure and in selected populations of patients with myocardial infarction without systolic heart failure [1, 24]. Its beneficial effects on cardiovascular system encompass blockade of beta-adrenoreceptor, reduction in sympathetic activity, antioxidant and anti-arrhythmic properties, positive actions on myocardial metabolism and protection of endothelium [25]. Patients did not show systolic heart failure with on average preserved LVEF in both groups. Some patients were on diltiazem instead of BB therapy. In contrast to $\mathrm{BB}$ which application has been associated with better long-term outcomes after HTx, the 
use of diltiazem did not show any advantage with regard to survival despite a similar reduction of heart frequencies as under BB [26]. Although it is known that diltiazem enhances $\mathrm{CsA}$ and tacrolimus concentrations in the blood and thus reduces the need of higher CsA and tacrolimus doses [27] and has positive effects on transplant vasculopathy [23] and cardiopulmonary performance [28], giving preference to BB therapy in a selected group of patients could be advantageous considering results from this study.

\section{Limitations of the study}

The present study has some limitations. The most important one is connected to its retrospective character and thus descriptive results. Additionally, the number of patients enrolled was relatively low. On the other hand, this statement relativizes itself when taking into consideration the monocentric design of the study. Furthermore, no differentiation into CD4 subtypes such as regulatory and effector T cells [29] was performed. However, the aim of this work was to search for simple predictors of mortality which can be determined easily and inexpensively in routine diagnostics. Finally, the findings from immune monitoring were completely available only at the last follow-up visit, so we cannot answer the question about the blood levels of immune cells as transplant vasculopathy was initially diagnosed or as respective organ rejections were detected and treated.

\section{Conclusions}

Taken together, the present study showed that lower CD4+ blood levels, systemic inflammation, organ rejections requiring therapies, early diagnosis of transplant vasculopathy and no use of BB therapy were associated with increased mortality in a long-term time course after HTx.

\section{Conflict of interest: None declared}

\section{References}

1. Ponikowski P, Voors A, Anker S, et al. ESC Scientific Document Group 2016. ESC Guidelines for the diagnosis and treatment of acute and chronic heart failure: The Task Force for the diagnosis and treatment of acute and chronic heart failure of the European Society of Cardiology (ESC). Developed with the special contribution of the Heart Failure Association (HFA) of the ESC. Eur Heart J. 2016; 37(27): 2129-2200, doi: 10.1093/eurhearti/ehw128.

2. Mehra MR, Canter CE, Hannan MM, et al. International Society for Heart Lung Transplantation (ISHLT) Infectious Diseases, Pediatric and Heart Failure and Transplantation Councils. The
2016 International Society for Heart Lung Transplantation listing criteria for heart transplantation: A 10-year update. J Heart Lung Transplant. 2016; 35(1): 1-23, doi: 10.1016/j.healun.2015.10.023, indexed in Pubmed: 26776864.

3. Zamperetti N, Bellomo R, Piccinni P, et al. Reflections on transplantation waiting lists. Lancet. 2011; 378(9791): 632-635, doi: 10.1016/S0140-6736(10)62343-4, indexed in Pubmed: 21752461.

4. Luckraz H, Goddard M, Charman SC, et al. Early mortality after cardiac transplantation: should we do better? J Heart Lung Transplant. 2005; 24(4): 401-405, doi: 10.1016/j.healun.2004.02.013, indexed in Pubmed: 15797739.

5. Foroutan F, Alba AC, Guyatt G, et al. Predictors of 1-year mortality in heart transplant recipients: a systematic review and meta-analysis. Heart. 2018; 104(2): 151-160, doi: 10.1136/ heartjnl-2017-311435, indexed in Pubmed: 28855271.

6. Tjang YS, van der Heijden GJ, Tenderich G, et al. Survival analysis in heart transplantation: results from an analysis of 1290 cases in a single center. Eur J Cardiothorac Surg. 2008; 33(5): 856-861, doi: 10.1016/j.ejcts.2008.02.014, indexed in Pubmed: 18356067.

7. Jung SH, Kim JJ, Choo SJ, et al. Long-term mortality in adult orthotopic heart transplant recipients. J Korean Med Sci. 2011; 26(5): 599-603, doi: 10.3346/jkms.2011.26.5.599, indexed in Pubmed: 21532848.

8. Jaramillo N, Segovia J, Gómez-Bueno M, et al. Characteristics of patients with survival longer than 20 years following heart transplantation. Rev Esp Cardiol (Engl Ed). 2013; 66(10): 797-802, doi: 10.1016/j.rec.2013.05.016, indexed in Pubmed: 24773860.

9. DeCampli WM, Luikart H, Hunt S, et al. Characteristics of patients surviving more than ten years after cardiac transplantation. J Thorac Cardiovasc Surg. 1995; 109(6): 1103-1114, doi: 10.1016/S0022-5223(95)70194-X, indexed in Pubmed: 7776675.

10. Bergenfeldt H, Lund LH, Stehlik J, et al. Time-dependent prognostic effects of recipient and donor age in adult heart transplantation. J Heart Lung Transplant. 2019; 38(2): 174-183, doi: 10.1016/j.healun.2018.10.003, indexed in Pubmed: 30502009.

11. Chaudhri B. Heart transplantation: new realities, challenges and developments - surgical perspectives. J Cardiol Curr Res. 2014; 1(3): 58-63, doi: 10.15406/jccr.2014.01.00013.

12. Stewart S, Winters GL, Fishbein MC, et al. Revision of the 1990 working formulation for the standardization of nomenclature in the diagnosis of heart rejection. J Heart Lung Transplant. 2005; 24(11): 1710-1720, doi: 10.1016/j.healun.2005.03.019, indexed in Pubmed: 16297770 .

13. Blanc B, Finch CA, Hallberg L, et al. Nutritional anaemias. Report of a WHO Scientific Group. WHO Tech Rep Ser. 1968; 405: 1-40.

14. Pietra BA, Wiseman A, Bolwerk A, et al. CD4 T cell-mediated cardiac allograft rejection requires donor but not host $\mathrm{MHC}$ class II. J Clin Invest. 2000; 106(8): 1003-1010, doi: 10.1172/JCI10467, indexed in Pubmed: 11032860.

15. Ducloux D, Carron PL, Rebibou JM, et al. CD4 lymphocytopenia as a risk factor for skin cancers in renal transplant recipients. Transplantation. 1998; 65(9): 1270-1272, doi: 10.1097/00007890199805150-00022, indexed in Pubmed: 9603180.

16. Kahan BD. Cyclosporine. N Engl J Med. 1989; 321: 1725-1738.

17. Sato Y, Yanagita M. Immunology of the ageing kidney. Nat Rev Nephrol. 2019; 15(10): 625-640, doi: 10.1038/s41581-019-0185-9, indexed in Pubmed: 31477915.

18. Yoon JW, Gollapudi S, Pahl MV, et al. Naïve and central memory T-cell lymphopenia in end-stage renal disease. Kidney Int. 2006; 70(2): 371-376, doi: 10.1038/sj.ki.5001550, indexed in Pubmed: 16738532 . 
19. Wallin EF, Hill DL, Linterman MA, et al. The Calcineurin Inhibitor Tacrolimus Specifically Suppresses Human T Follicular Helper Cells. Front Immunol. 2018; 9: 1184, doi: 10.3389/ fimmu.2018.01184, indexed in Pubmed: 29904381.

20. Li Y, Zhong X, Cheng G, et al. Hs-CRP and all-cause, cardiovascular, and cancer mortality risk: A meta-analysis. Atherosclerosis. 2017; 259: 75-82, doi: 10.1016/j.atherosclerosis.2017.02.003.

21. Chapman JR, Webster AC, Wong G. Cancer in the transplant recipient. Cold Spring Harb Perspect Med. 2013; 3(7): a015677, doi: 10.1101/cshperspect.a015677, indexed in Pubmed: 23818517.

22. Ducloux D, Challier B, Saas P, et al. CD4 cell lymphopenia and atherosclerosis in renal transplant recipients. J Am Soc Nephrol. 2003; 14(3): 767-772, doi: 10.1097/01.asn.0000048718.43419.44, indexed in Pubmed: 12595514.

23. Gupta S. Drugs for the prevention and treatment of cardiac allograft vasculopathy. Cardiol Pharmacol. 2014; 3: 2.

24. Ibanez B, James S, Agewall S, et al. ESC Scientific Document Group. 2017 ESC Guidelines for the management of acute myocardial infarction in patients presenting with ST-segment elevation: The Task Force for the management of acute myocardial infarction in patients presenting with ST-segment elevation of the European
Society of Cardiology (ESC). Eur Heart J. 2018; 39(2): 119-177, doi: 10.1093/eurhearti/ehx393, indexed in Pubmed: 28886621.

25. Rehsia NS, Dhalla NS. Mechanisms of the beneficial effects of beta-adrenoceptor antagonists in congestive heart failure. Exp Clin Cardiol. 2010; 15(4): e86-e95, indexed in Pubmed: 21264074.

26. Ciarka A, Lund LH, Van Cleemput J, et al. Effect of Heart Rate and Use of Beta Blockers on Mortality After Heart Transplantation. Am J Cardiol. 2016; 118(12): 1916-1921, doi: 10.1016/j. amjcard.2016.08.084, indexed in Pubmed: 27743576.

27. Page RL, Miller GG, Lindenfeld J. Drug therapy in the heart transplant recipient: part IV: drug-drug interactions. Circulation. 2005; 111(2): 230-239, doi: 10.1161/01.CIR.0000151805.86933.35, indexed in Pubmed: 15657387.

28. Varnado S, Peled-Potashnik Y, Huntsberry A, et al. Effect of diltiazem on exercise capacity after heart transplantation. Clin Transplant. 2017; 31(8), doi: 10.1111/ctr.12997, indexed in Pubmed: 28477381.

29. Hoerning A, Köhler S, Jun C, et al. Cyclosporin but not everolimus inhibits chemokine receptor expression on $\mathrm{CD} 4+\mathrm{T}$ cell subsets circulating in the peripheral blood of renal transplant recipients. Clin Exp Immunol. 2012; 168(2): 251-259, doi: 10.1111/j.13652249.2012.04571.x, indexed in Pubmed: 22471287. 